\title{
Energy Efficient and Quality-of-Service Aware Routing using Underwater Wireless Sensor Networks
}

\author{
P. Sathya ${ }^{1}$, P. Sengottuvelan ${ }^{2}$ \\ Ph.D Research Scholar ${ }^{1}$, Associate Professor ${ }^{2}$, \\ Department of Computer Science, Periyar University PG Extension Centre, Dharmapuri, Tamilnadu, India ${ }^{1,2}$
}

\begin{abstract}
In current years, there has been an increasing attention in Underwater wireless sensor networks (UWSNs). Underwater sensor networks (USNs) can be applied for many various purposes. To address the routing issue, the Cuckoo Search Optimization Algorithm with Energy Efficient and QOS Aware (CSOA-EQ) based routing methods have been proposed in this chapter. Every application is important in its own right, but some of them can help improve sea investigation to meet a variety of underwater applications, such as a catastrophic event alert system (such as torrent and seismic monitoring), supported navigation, oceanographic data collection, and underwater surveillance, ecological applications (such as the nature of organic water and contamination monitoring), modern applications (such as marine investigation), and so on. For example, sensors can assess specific metrics, such as base intensity and securing pressure, to monitor the auxiliary nature of the securing environment in offshore engineering applications. UASNs have also improved our understanding of underwater environments, such as climate change, underwater creature life, and the number of inhabitants in coral reefs.
\end{abstract}

Keywords-Underwater wireless sensor networks (UWSNs); QOS aware (CSOA-EQ); underwater environments

\section{INTRODUCTION}

Sensor Networks have emerged as a potential examination topic this year. In these types of networks, the routing issue is a critical component that must be handled in order to extend the life of the organisation. Because of the number of sensor nodes in the organisation, routing becomes increasingly unpredictable as the size of the organisation grows [1]. Sensor nodes in Wireless Sensor Networks are highly reliant on memory, processing power, and battery life.

Surface buoys operational with GPS can acquire their regions, as seen in Fig. 1. The numeral of beacon nodes be far lower than the amount of obscure nodes. Because beacon nodes have more energy and a communication range of roughly 200 metres, they can legitimately communicate with buoys and have more neighbours. Furthermore, compared to hidden nodes, beacon nodes have more equipment assets and superior figuring capacity, allowing them to do more. The obscure nodes are slightly less expensive, and they aren't required to waste energy [2]. An obscure hub's communication radius is roughly 100 metres, and it is unable to officially communicate with the buoys. It can normally only associate with its immediate (usually one-jump) neighbors [3]. The obscure nodes can achieve nearby positioning to successfully participate in the organization exercises thanks to local data exchange among themselves and nearby beacon nodes.
Optimization is everywhere, so there are many applications for this paradigm [4]. In practically all technical and industrial applications, we are continually looking for ways to improve anything, whether it is to minimize cost and energy consumption or to boost benefit, yield, execution, and effectiveness [5]. Because resources, time, and money are all limited in practice, optimization is undoubtedly more important. Because most real-world applications have clearly more complex variables and parameters influencing how the framework functions, making the greatest use of any given assets needs a paradigm shift in logical reasoning. The essential components of the optimization cycle for each optimization challenge are the optimization algorithm, an effective numerical test system, and a realistic-portrayal of the physical cycles we want to illustrate and optimize [6]. After we have a good model, the general computation costs are dictated by the optimization strategies used for search and the numerical solver utilized for simulation. To address the routing issue, Cuckoo Search Optimization Algorithm with Energy Efficient and QOS Aware (CSOA-EQ) based routing methods have been proposed [7]. A wireless sensor network (WSN) is made up of self-contained sensor nodes. These sensors are extremely small. They are widely disseminated in large numbers [8]. These sensor nodes are intelligent and successful, providing a very wonderful and adaptive network where regular wired and wireless networks are unable to deliver. WSN is used in a variety of engineering applications, including monitoring unfenced far margins, terrorist development in high-altitude backwoods territories, and LPG pipe lines put in deep water. The sensor node detects or monitors movement or functions in a network region or area and delivers information to the base station.

A QoS aware routing provides optimality when sensor nodes are transmitted in underwater sensing applications, such as monitoring LPG pipelines [9]. As a result, this research also focuses on QoS-aware routing. The compromise between network QoS assurance and network lifetime is a fundamental issue in QoS aware routing, i.e., the presentation of QoS aware routing and the implementation of Energy efficient routing are diametrically opposed. The main goal is to maintain the energy-efficient and QoS-aware routing running smoothly. This chapter describes WSN execution and compares it to ACO and PSO's suggested CSOA-EQ execution in terms of normal number of ways, energy consumption, and normal parcel delay [10]. The proposed CSOA-execution EQ's is considerably enhanced when compared to ACO and PSO. 


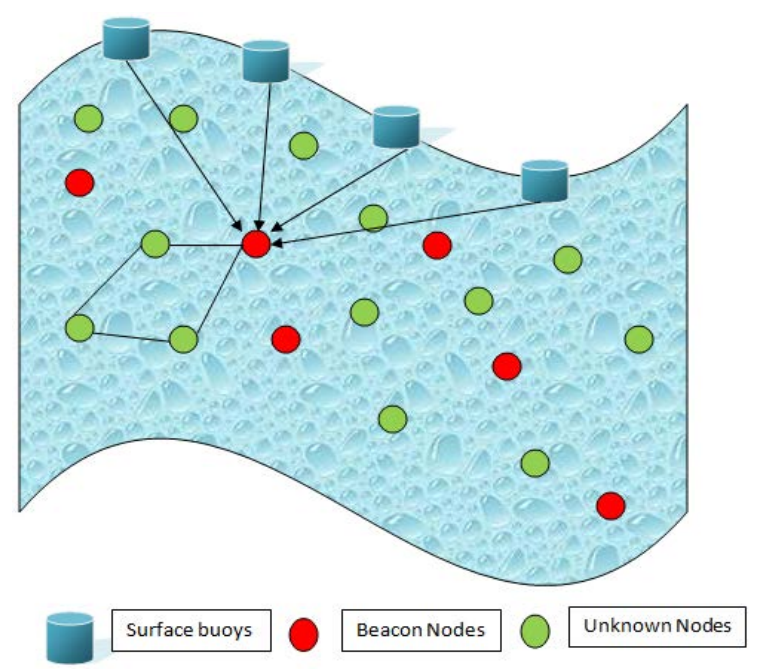

Fig. 1. Schematic Diagram of UWSN.

\section{LiterATURE REVIEW}

Sudip Misra, Anudipa Mondal, and Ayan Mondal (2019) introduced DATUM, a game theory-based dynamic topology control approach for enhancing throughput and network lifetime in UWMSNs within the sight of interactive media sensor nodes with the least network latency [1]. This author uses a cooperation game theoretic approach to select the best combination of methods for limiting postponement and amplifying throughput, as well as the best transmission power for extending network lifetime. Luis M. Pessoa, Cândido Duarte, Henrique M. Salgado, Vasco Correia, Bruno Ferreira, Nuno A. Cruz, and Anibal Matos (2019) evaluate the longterm deployment feasibility of a large-scale network of abandoned underwater sensors, with power provided by autonomous underwater vehicles (AUVs) in periodic visits [2]. They conduct a versatile analysis to determine the size of network that can be supported by a single AUV, both in terms of total number of sensors and partition separation between sensors.

\section{EXISTING METHOdOLOGIES}

\section{A. Ant Colony Optimization Algorithm}

The field of "Ant Algorithm" investigates how models derived from observations of real ants' behavior stimulate the development of novel algorithms for solving distributed control problems via optimization [11]. The basic idea is that self-organizing standards, which take into account the profoundly co-ordinate behavior of actual ants, may be utilized to co-ordinate populations of artificial agents working together to solve computing problems. Various forms of ant algorithms, including as seeking, division of labour, brood arranging, and co-employable vehicle, have been enlivened by a few distinct aspects of ant province behavior. "One of the better examples of ant algorithms is "Ant Colony Optimization (ACO)"[12]. Because the forward ants in the ACO strategy are sent to no specific destination node in the essential algorithm, sensor nodes must communicate with one another and every node's routing tables must contain the IDs of all sensor nodes in the neighbourhood as well as the journalist levels of the pheromone trail, making the ACO strategy more energy efficient. In big networks, this can be an issue because nodes would require a lot of memory to save all of the data about the neighbourhood.

\section{B. Particle Swarm Optimization}

Molecule Swarm Optimization (PSO) was developed by Kennedy and Eberhart in 1995, based on a range of natural behaviours such as fish and flying creature learning. PSO has since sparked a plethora of new interests and structures an exhilarating, ever-expanding study topic known as swarm insight. This method searches the spaces of a target work by changing the trajectories of individual agents, termed particles, in a quasi-stochastic manner as piecewise ways determined by positional vectors [13].Particle swarm optimization (PSO) mathematical expression is followed[14]. Assuming a Dimensional search space, $m$ particles form a group. The position of the particle I in the search space is $x_{i}$ and Vector is $x_{i}=\left(x_{i 1}, x_{i 2}, L x_{i D}\right)^{T}$ and the flying speed is $V_{i}=\left(v_{i 1}, v_{i 2}, L v_{i D}\right)^{T}$. The individual extremum of particle I is $P_{i}=\left(P_{g 1}, P_{g 2}, L, P_{g D}\right)^{T}$. In the formula $g$ is the number of fitness optimum in the group [13]. The particles are iterative operation according to the following formula (3) and (4) and schematic diagram is shown in Fig. 4.

$v_{i d}^{k+1}=w v_{i d}^{k}+c_{1} \cdot r_{1} \cdot\left(p_{i d}^{k}-x_{i d}^{k}\right)+c_{2} \cdot r_{2} \cdot\left(g_{i d}^{k}-x_{i d}^{k}\right)$

$x_{i d}^{k+1}=x_{i d}^{k}+v_{i d}^{k+1}$

$\omega$ is non-unconstructive number, called the interia weight (intertia weight), Its role is not to adjust the algorithm of global and local search ability of balance. $\mathrm{I}=1,2, \ldots, \mathrm{m}$ $\mathrm{d}=1,2, \ldots, \mathrm{D}$. Acceleration constant $c_{1}$ and $c_{2}$ (acceleration constant) is a non negative; $r_{1}$ and $r_{2}$ is a random number between. $v_{i d} \in\left[-v_{\max }, v_{\max }\right] ; v_{\max }$ is a constant, setting by the user; $\mathrm{k}=1,2, \ldots$ is the number of iterations [15].

\section{PRoposed Method}

\section{A. Network Architecture}

First, we'll go through the network architecture employed in this chapter. The sensor network in this scenario is a Big Network (BN), with the water's surface as the top surface and the seabed as the bottom surface. This underwater sensor network is organized into miniature crow nests, as seen in Fig. 2 (CNs). Fig. 1 shows the network architecture of the suggested model.

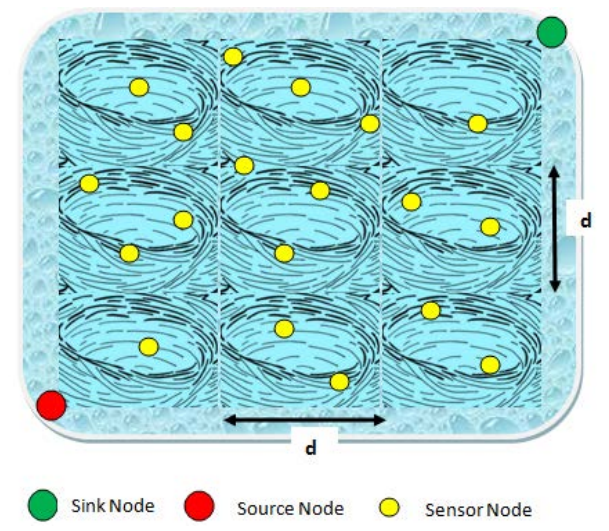

Fig. 2. Network Architecture of the Proposed Model. 
A source node should be at the lower left corner of the BN, whereas a sink node should be in the upper right corner. Allow $\mathrm{Na}$ sensor nodes equipped with audio modems to communicate with sonobuoys positioned at arbitrary depths. These nodes are all stagnant and do not have any water flowing through them. Each node in the network has the same main energy, transmission power, and range, as well as symmetric interactions between nodes. The sink node is assumed to also be equipped with an offshore level sonobuoy, and that each node is aware of both its own and the sink node's location. Furthermore, as seen in Fig. 2, there are several nodes within a CN. Furthermore, as seen in Fig. 2, there are many nodes within a $\mathrm{CN}$ that store the $\mathrm{CN}$ that they have a place with. The length of the $\mathrm{CN}$ is commonly referred to as $\mathrm{d}$, and it is entirely dependent on $\mathrm{r}$, which is the sensor node exchange radius. Similarly, the duty cycle technique assumes that each nodes condition varies independently of the state of the others. Because each node is awake for a short period of time before going to sleep for the remainder of the time, this is the simplest type of obligation cycle. There is no need for global synchronization because each sensor node can maintain track of its own and the sink node's locations.

\section{B. CSOA-EQ Algorithm}

Similarly, because the routing database does not exist, no RAM is utilized to save the path. Additionally, the multiplerouting method will be combined with geo-routing to improve the reliability of packets being received efficiently, and packets will be dispatched from many routes at the same time. Furthermore, the duty cycle instrument allows the nodes to rest occasionally to conserve energy while no data is being transmitted. The network is said to be a Big Network separated into little crow homes. In the proposed technique; each sensor node can communicate directly with all of its vertex, edge, and surface adjacent nodes. A CN within a $\mathrm{BN}$, as seen in Fig. 3, node $\mathrm{u}$, is an outstanding illustration of this type of CN.

In Fig. 4, a flowchart of the proposed method is exposed.

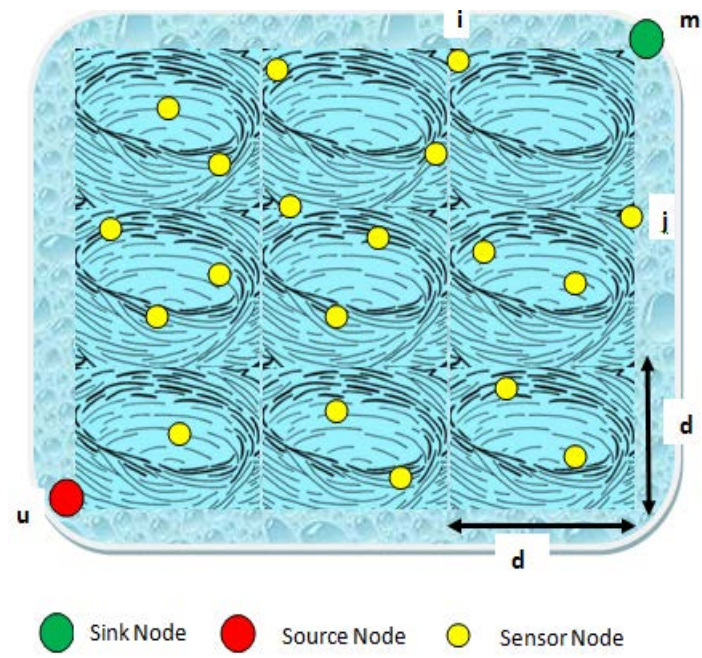

Fig. 3. Relation between $r$ and $d$ in the Network.

\section{Proposed CSOA-EQ Algorithm}

Similarly, the routing database does not exist, so no memory is used in the process of storing the path. Furthermore, the multiple-routing process will be used with geo-routing to increase the reliability of packets being received effectively, and packets will be dispatched off the destination at the same time from numerous routes. In addition, the duty cycle instrument allows the nodes to occasionally rest in order to save energy while no data is being delivered. It's thought that the network is a Big Network divided into small crow homes. To prevent packet flooding, each node's diffusion radius is defined, and every node can only transfer data within this radius. Furthermore, in the suggested algorithm, node selection is settled in a later step depending on the node's reasonableness.

Every sensor node in the proposed algorithm can communicate directly with all of its vertex-adjacent, edgeadjacent, and surface-adjacent nodes. It should be noted that a $\mathrm{CN}$ within the BN, as shown in Fig. 2, is an excellent example of such a CN. Similarly, node $\mathrm{m}$ is the farthest node with whom u can have a direct conversation. As a result, the Base Exchange radius of node $\mathrm{u}$ is determined by the Euclidean distance between $\mathrm{u}$ and $\mathrm{m}$.

As a result, the following equation determines the relationship between the length of the CN edge (d) and the transfer radius of the sensor nodes (r)

$$
(2 d)^{2}+(2 d)^{2}+(2 d)^{2}=r^{2} d=\frac{r}{\sqrt{12}}
$$

- First, as shown in Fig. 1, Na nodes are haphazardly spread at various depths of $\mathrm{H}$ in BN space to depict the suggested technique. Based on the measure of $d$, the $\mathrm{BN}$ is then partitioned into various CNs. Using rangebased or without range localization techniques; each node should also know its own location and the location of the sink node. Fig. 5 illustrates this. Furthermore, each node must be aware of which CN it belongs to. The fact that each of these nodes has the same fundamental energy is crucial. The routing cycle begins in two steps after the fundamental design of the nodes:

- Stage 1: The initial phase of the routing for the source node $\mathrm{u}$ is discovering all of the node's nearby Crow Nest (CN) subunits. A CN can have vertex-adjacent, edge-adjacent, and surface-adjacent neighbours, as previously stated. We select CNs that are closer to the sink than the current Crow Nest $(\mathrm{CN})$ and have at least one awake node from all nearby CNs. This is to prevent the course from being bypassed in vain and the sink way from being escaped. The nodes in these CNs are then recorded and assessed as possibilities for the next stage. Following the shaping of a large number of applicant nodes, the second routing stage begins with the CSOA-EQ algorithm, as shown below. 


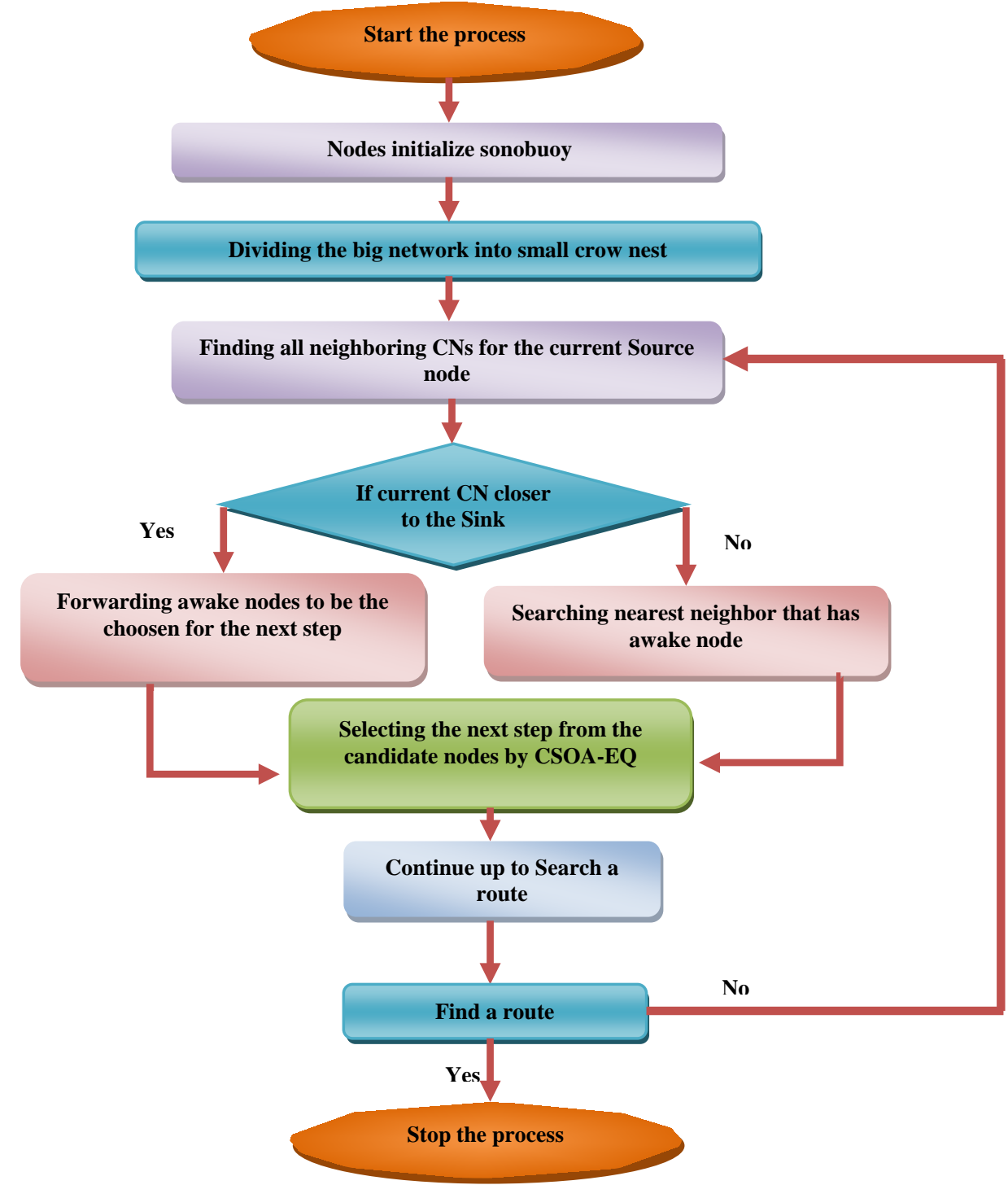

Fig. 4. Flowchart of the Proposed Method.

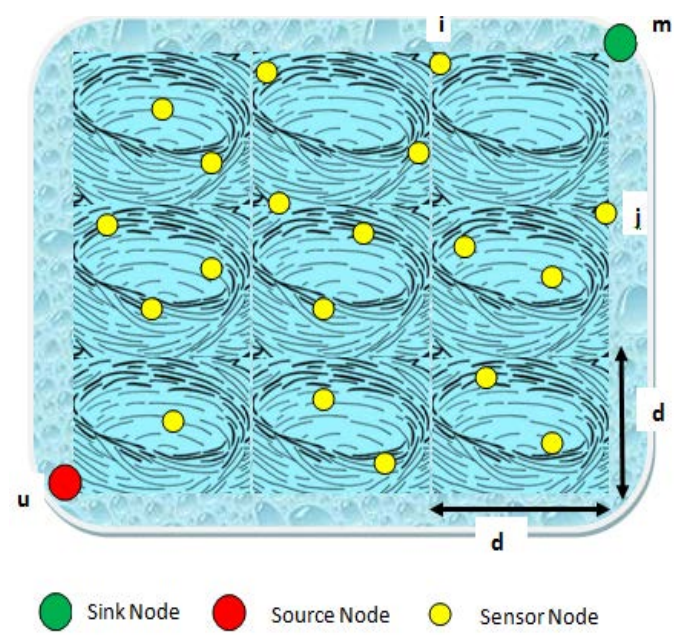

Fig. 5. Relation between $r$ and $d$ in the Network. 
- Stage 2: First, mother cuckoos are horizontally distributed across the search field using the Cuckoo Search Optimization Algorithm. The following advancements are then carried out in order to achieve the optimal precision in optimization.

- Step 1: A random number of eggs is allotted to each mother cuckoo. The equation then determines the Egg Laying Radius (ELR) of each bird in relation to this number.

$\mathrm{ELR}=\beta \times \frac{\text { Number of current cuckoo'seggs }}{\text { Total number of eggs }} \times\left(\right.$ var $_{\text {hi }}-$ var $\left._{\text {low }}\right)$

Where is a positive integer that represents the maximum ELR value. It's that each bird can only lay eggs within a certain radius.

- Step 2: Mother cuckoos lay their eggs in this area. After the laying process, the profit value of all mother cuckoos and egg is calculated using the cost function (described below). Then, in order to keep the cuckoo population under control, we destroy eggs with a lower yield. The laying process is completed at this point, and the eggs are grown and matured over time.

- Stage 2: This is where mother cuckoos lay their eggs. The benefit estimation of all mother cuckoos and eggs is calculated after the laying cycle using the cost work given below. To regulate the most extreme population of cuckoos, we kill eggs with a lower yield at that time. Near the end of this stage, the laying cycle is completed and the eggs are formed and developed.

- Stage 3: The presence of young cuckoos creates a new population of cuckoos. The algorithm's current mothers are young cuckoos. The cuckoos' dwelling areas are then grouped using the K-mean grouping technique. Because the search field and number of optimization are not large in this chapter, $\mathrm{K}$ is assumed to be 1 .

- Stage 4: Following bunching, the benefit of each group is calculated, and the bunch with the highest benefit is presented as the best place for cuckoo relocation. In the same way, the cuckoo with the highest profit in this group is chosen as the worldwide ideal.

- Stage 5: If the algorithm arrives at the ideal combination, it stops; in any case, these means are rehashed. The issue in the CSOA-EQ algorithm is the closeness of the cuckoo's populace to one another. In the event that the cuckoo populace is near the greatness of the intermingling coefficient indicated in the algorithm, the algorithm has arrived at its assembly.

It ought to be noticed that CSOA-EQ yield is the global optimal arrangement which is accomplished by adjusting it to the neighboring node with the most elevated net revenue. The above advances proceed until there are no different nodes or CNs that gives routing conditions.

The following bounce node is best picked by the CSOAEQ algorithm in this chapter based on the node with the maximum energy() in the objective CN. The distance between the current node and the chosen node, the method misfortune based on the propagation delay, and the measure of the current node's residual energy are all factors that influence this decision. Regardless of the standards, the energy required to send, rest of the node's energy when data is shipped off this node, and the node's underlying energy are all taken into account in this cost calculation Similarly, because exponential-sine capabilities are ones in which minor changes in parameters can result in dramatic changes in the capacity's implications, the cost work for selecting the next bounce node is as follows:

$\grave{v}_{\mathrm{ij}}=\frac{\mu}{\mathrm{D}}+\frac{\mathrm{K}}{\mathrm{L}}+\tau \mathrm{C}_{\mathrm{ij}}+\zeta \mathrm{E}_{\text {resi }}$

In Eq. (3), $\mu, \kappa, \tau, \zeta$, they're constant coefficients with a sum of 1 that's utilized to manage the weight in the cost function. Cij is also defined by.

$C=E_{i j}^{r e m} \exp \left[1 / \sin \left(\pi-\frac{\frac{\pi}{2} E_{i j}^{r e m}}{E_{0}}\right)\right]$

In the above relation $E_{i j}^{r e m}$ is the remaining energy of node I if sent to node $\mathrm{j}, \mathrm{E}_{\mathrm{ij}}^{\mathrm{rem}}$ is the current remaining energy of node $i$ and $E_{0}$ is the initial energy of node $i$. Accordingly, based on the relation, the optimal selection of the next step for node I through CSOA-EQ algorithm based on the node with the highest energy in the crow nest is in the crow nest is made by.

$\mathrm{J}=\operatorname{Arg} \max _{\mathrm{j} \in\left\{\mathrm{SC}, \mathrm{N}_{\mathrm{d}}\right\}}\left(\omega_{\mathrm{ij}}\right)$

Here, $N_{d}=\alpha N_{a}$ is a set of all awake nodes in the network according to the duty-cycle mechanism, Where nodes and $0<$ $\alpha \leq 1$ is the duty-cycle parameter. It is worth nothing that the larger $\alpha$ becomes, the more paths there are for the next selection, and so the network can route information from the source to the sink through different paths.

\section{Time Complexity}

As shown in Algorithm 2, CSOA-EQ for temporal complexity is made up of five key phases. Here's a quick rundown of each stage and its associated time complexity:

- Step 1: The first stage, according to Equation, is to calculate the dependability constraint score (6). The temporal complexity of this step can be estimated as follows: $\mathrm{O}(|\mathrm{T}||\mathrm{AC}|)$.

$\sum_{\forall c_{j} \in A_{C}} Y_{i, j} X_{j} \geq K ; \forall \mathrm{v}_{i} \in T$

- Step 2: The second step is to calculate the time constraint score using Equation (7).This step takes a long time: $\mathrm{O}(|\mathrm{T}||\mathrm{AC}|)$.

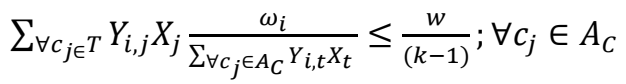

- Step 3: The third step is to calculate the maximum distance between sensors and controllers using Equation (8).the time complexity of this step is: $O$ (|T||AC|).

$L_{\mathrm{v}_{i}}^{*}=\max _{\forall c_{j} \in A_{C}}\left\{Y_{i, j} X_{j} l^{*}\left(\mathrm{v}_{i}, C_{j}\right)\right\}$ 
- $\quad$ Step 4: The fourth phase entails sorting all of the eggs into fitness categories. The temporal complexity of this phase can be represented as $\mathrm{O}$ (no of eggs) $\times$ Npop log Npop.

- Step 5: The fifth stage entails sorting mature cuckoos based on their fitness values, which is a lengthy process: O Npop log Npo.

Hence,the time complexity can be written as O Npop log Npop $+|\mathrm{T}||\mathrm{AC}|$. Furthermore, while increasing network size does not result in a significant change in Npop, it can be concluded that $|\mathrm{T} \| \mathrm{AC}|$ has upper hand on Npop log Npop. As a result, Cuckoo-overall PC's time complexity is: $\mathrm{O}$ (|T||AC).

\section{EXPERIMENTAL RESULTS}

\section{A. Number of Sensor Nodes on Average Number of Paths Found}

Table I compares the number of sensor nodes to the average number of pathways discovered, which explains the ACO, PSO, and CSOA-EQ results. When the existing approaches and the new CSOA-EQ are compared, the proposed method produces better results (Fig. 6).

TABLE I. Number of Sensor Nodes on AVerage Number of Paths FOUND

\begin{tabular}{|l|l|l|l|}
\hline $\begin{array}{l}\text { Number of sensor } \\
\text { nodes }\end{array}$ & ACO & PSO & $\begin{array}{l}\text { Proposed } \\
\text { CSOA-EQ }\end{array}$ \\
\hline 200 & 1.255 & 1.468 & 1.861 \\
\hline 400 & 2.216 & 2.365 & 2.632 \\
\hline 600 & 2.945 & 3.014 & 3.265 \\
\hline 800 & 3.465 & 3.984 & 4.147 \\
\hline 1000 & 4.875 & 4.971 & 5.852 \\
\hline
\end{tabular}

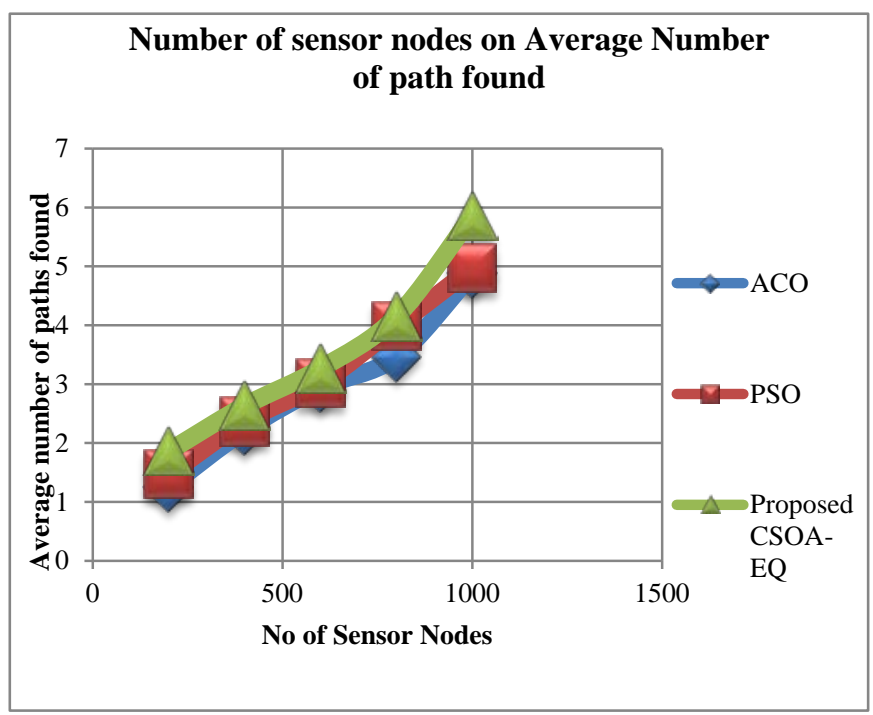

Fig. 6. Sensor Nodes on Average Number of Paths Found.

The evaluation table of Number of sensor nodes on average number of paths detected explains the ACO, PSO, and recommended CSOA-EQ values. When the results of existing approaches and the proposed method are compared, the suggested CSOA-EQ method comes out on top. We can also observe that as the number of nodes grows, so does the number of paths detected, resulting in enhanced network dependability and speed in identifying the path within the network.

\section{B. Number of Sensor Nodes on Energy Consumption}

Table II shows a comparison table of the number of sensor nodes on the average number of pathways identified, which explains the ACO, PSO, and CSOA-EQ results. When comparing existing approaches to the proposed CSOA-EQ, Fig. 7 shows that the proposed CSOA-EQ uses less energy.

The comparison table of number of sensor nodes on average number of paths detected explains the ACO, PSO, and proposed CSOA-EQ values. When comparing the existing and suggested approaches, the proposed CSOA uses the least amount of energy.

\section{Average Packet Delay}

Table III provides the Average Packet Delay Comparison Table, which illustrates the differences in ACO, PSO, and CSOA-EQ values. Fig. 8 shows that when existing approaches are compared to the proposed CSOA-EQ, the suggested method produces better results.

The ACO, PSO, and suggested CSOA-EQ values are explained in the Comparison chart of Number of sensor nodes on average packet latency. When comparing the outcomes of the existing approaches and the proposed method, the suggested CSOA-EQ provides better results.

TABLE II. NUMBER OF SENSOR NODES ON ENERGY CONSUMPTION

\begin{tabular}{|l|l|l|l|}
\hline $\begin{array}{l}\text { Number of sensor } \\
\text { nodes }\end{array}$ & ACO & PSO & $\begin{array}{l}\text { Proposed } \\
\text { CSOA-EQ }\end{array}$ \\
\hline 200 & 9.024 & 8.802 & 6.789 \\
\hline 400 & 8.632 & 7.744 & 5.065 \\
\hline 600 & 8.125 & 7.062 & 4.732 \\
\hline 800 & 7.851 & 6.745 & 4.487 \\
\hline 1000 & 6.974 & 6.196 & 2.954 \\
\hline
\end{tabular}

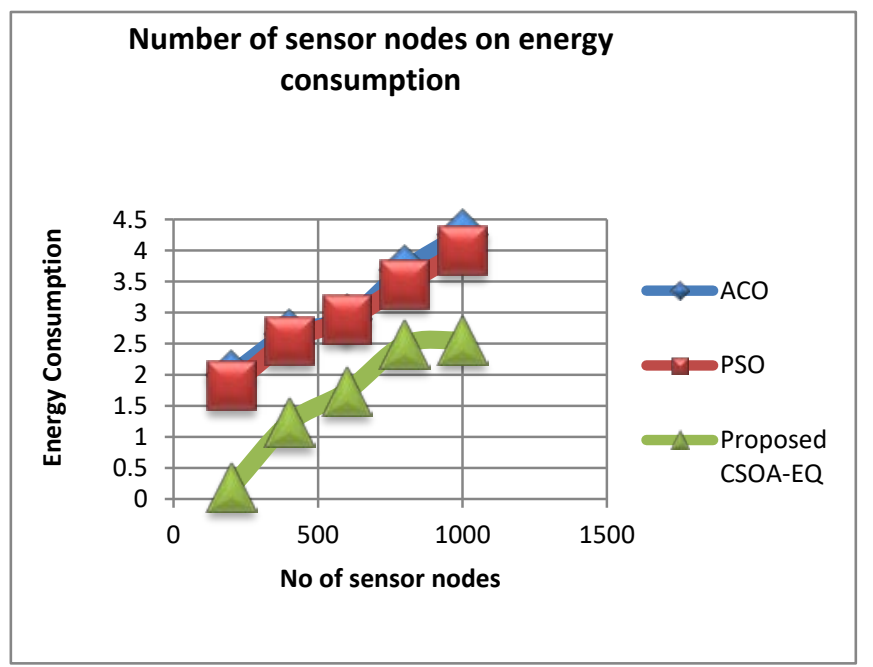

Fig. 7. Sensor Nodes on Average Number of Paths Found. 
TABLE III. AVERAge PACKET DELAY

\begin{tabular}{|l|l|l|l|}
\hline $\begin{array}{l}\text { Number of sensor } \\
\text { nodes }\end{array}$ & ACO & PSO & $\begin{array}{l}\text { Proposed } \\
\text { CSOA-EQ }\end{array}$ \\
\hline 200 & 1.999 & 1.821 & 0.189 \\
\hline 400 & 2.654 & 2.544 & 1.225 \\
\hline 600 & 2.891 & 2.892 & 1.732 \\
\hline 800 & 3.684 & 3.456 & 2.487 \\
\hline 1000 & 4.258 & 3.996 & 2.454 \\
\hline
\end{tabular}

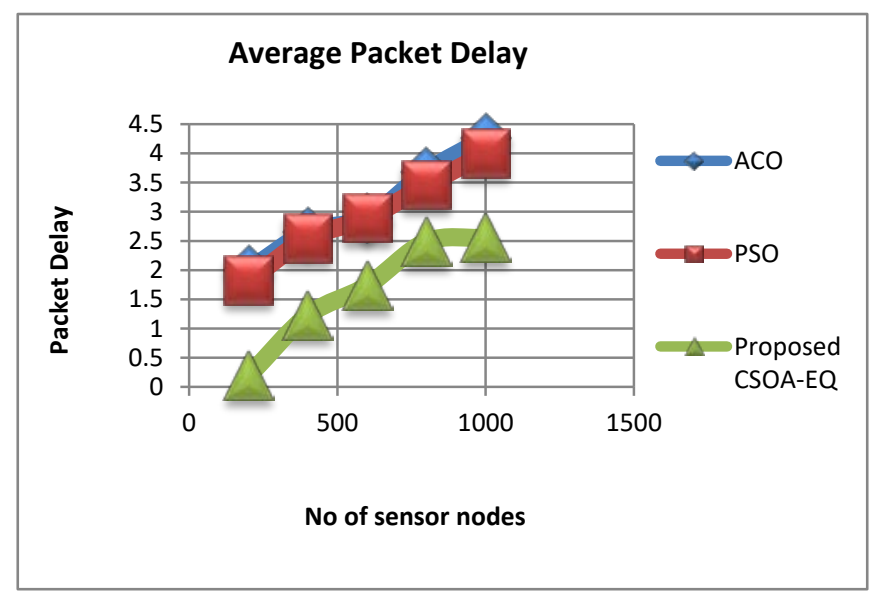

Fig. 8. Sensor Nodes on Average Packet Delay.

\section{CONCLUSION}

Cuckoo Search Optimization Algorithm with Energy Efficient and QOS Aware (CSOA-EQ) based routing methods have been proposed in this research to address the routing issue while attempting to handle these constraints. The proposed approach is also utilized to boost the likelihood of items being delivered successfully. By using duty cycle approach, nodes are also placed to sleep on a regular basis to save energy while no data is delivered. By introducing a differential equation in the CSOA-EQ algorithm, path selecting in the proposed technique is performed based on energy utilization and residual energy of the current node. When designing a network path for a WSN, fuel efficiency is a crucial consideration. The effectiveness of CSOA-EQ is appropriate in many application areas, as evidenced by its use in WSN routing issues. In this paper, we present a routing technique just on Cuckoo Search Optimization Algorithm with Energy Efficient and QOS Aware (CSOA-EQ), and also a probability of route selection based on pheromone and residual energy. We established that the proposed strategy is more energy efficient through simulated results. As part of future research, the CSOA-EQ algorithm will be used to increase network longevity, energy usage, and average packet latency.

\section{REFERENCES}

[1] SudipMisra, AnudipaMondal, and AyanMondal (2019), "DATUM: Dynamic Topology Control for Underwater Wireless Multimedia Sensor Networks", Electronic ISSN: 1558-2612, DOI: 10.1109/WCNC.2019.8885632, IEEE.
[2] Luís M. Pessoa, Cândido Duarte, Henrique M. Salgado, Vasco Correia, Bruno Ferreira, Nuno A. Cruz and Anibal Matos (2019), "Design of an underwater sensor network perpetually powered from AUVs", Electronic ISBN: 978-1-7281-1450-7, 10.1109/OCEANSE.2019.8867273, IEEE.

[3] Sai Wang and Yoan Shin (2019), “3D-Deployment of Magnetic Induction Relays in Underwater Sensor Networks", DOI: 10.1109/ICOIN.2019.8718105, Electronic ISBN: 978-1-5386-8350-7, IEEE, pp.222-226.

[4] Zhenghao Xi, XiuKan, Le Cao, Huaping Liu, GunasekaranManogaran, George Mastorakis, Constandinos and X. Mavromoustakis (2019), "Research on Underwater Wireless Sensor Network and MAC Protocol and Location Algorithm”, DOI: 10.1109/ACCESS.2019.2901375, Electronic ISSN: 2169-3536, IEEE.

[5] Judith Santana Abril, Graciela Santana Sosa, and Javier Sosa (2019), "Design of a Wireless Sensor Network for Oceanic Floating Cages in Aquaculture”, DOI: 10.1109/MWSCAS.2019.8885256, Electronic ISBN: 978-1-7281-2788-0, IEEE,pp.977-980.

[6] AliyuDala, TughrulArslan, and Imran Saied (2019), "Design of a Triangular Slotted Parasitic Yagi-Uda Antenna for Underwater Linear Sensor Network," IEEE, DOI: 10.1109/comite.2019.8733431, ISBN: 978-1-5386-9337-7.

[7] En Cheng, Longhao Wu, Fei Yuan, Chuanxian Gao, Jinwang Yi (2019), "Node selection algorithm for underwater acoustic sensor network based on particle swarm optimization," IEEE Electronic ISSN: 2169-3536, DOI: 10.1109/ACCESS.2019.2952169.

[8] MohamadMortadaa, AbdallahMakhoula, ChadyAbouJaoudeb, Hassan Harbb, and David Laiymani (2019), "A Distributed Processing Technique for Sensor Data Applied to Underwater Sensor Networks," IEEE.pp.979-984, DOI: 10.1109/IWCMC.2019.8766742, Electronic ISBN: 978-1-5386-7747-6.

[9] Gang Zhao, Yaxu Li, and Lina Zhang (2019)SSEEP: State-Switchable Energy-Conserving Routing Protocol for Heterogeneous Wireless Sensor Networks, DOI: 10.1109/ICEIEC.2019.8784570, Electronic ISBN: 978-1-7281-1190-2, pp.685-689.

[10] ShreemaShetty, Radhika M Pai\&Manohara M. M. Pai (2018), “Design and implementation of aquaculture resource planning using underwater sensor wireless network", ISSN: (Print) 2331-1916 (Online) 6 https://doi.org/10.1080/23311916.2018.1542576. Cogent Engineering (2018).

[11] NadeemJavaid, HammadMaqsood, Abdul Wadood, IftikharAzimNiaz, Ahmad Almogren, AtifAlamri, and ManzoorIlahi (2017), "A Localization Based Cooperative Routing Protocol for Underwater Wireless Sensor Networks", https://doi.org/10.1155/2017/7954175, Hindawi.pp.1-39.

[12] KumuduMunasinghe, Mohammed Aseeri, Sultan Almorqi, Md. FarhadHossain, MusbihaBinteWali, and Abbas Jamalipour (2019), "EM-Based High Speed Wireless Sensor Networks for Underwater Surveillance and Target Tracking”, https://doi.org/10.1155/2017/6731204, Hindawi Journal of Sensors. Pp.1-14.

[13] YishanSu ,YongpengZuo, Zhigang Jin , and Xiaomei Fu (2019), "OSPG-MAC: An OFDMA-Based Subcarrier Pregrouping MAC Protocol for Underwater Acoustic Wireless Sensor Networks", https://doi.org/10.1155/2019/4965231, Article ID 4965231, Hindawi Journal of Sensors.pp.1-12.

[14] Pei-Hsuan Tsai, Rong-Guei Tsai, and Shiuan-Shiang Wang (2017), "Hybrid Localization Approach for Underwater Sensor Networks", https://doi.org/10.1155/2017/5768651, Article ID 5768651, Hindawi Journal of Sensors. Pp.1-13.

[15] Fang Zhu and Junfang Wei (2018), “An Energy Efficient Routing Protocol Based on Layers and Unequal Clusters in Underwater Wireless Sensor Networks”, Article ID 5835730, https://doi.org/10.1155/2018/5835730, Hindawi Journal of Sensors. pp.1-11. 